\title{
Bereitstellung synthetischer Schwungmasse durch Wasserkraftwerke
}

\author{
R. Schürhuber, A. Lechner, W. Gawlik
}

\begin{abstract}
Die ausreichende Verfügbarkeit der für die Systemstabilität wichtigen Momentanreserve, aktuell bereitgestellt durch die Schwungmasse rotierender Maschinen, wird in einem zunehmend von erneuerbarer Einspeisung über Wechselrichter geprägten Netz immer wichtiger. In diesem Kontext werden die Bereitstellung und der Abruf von synthetischer Schwungmasse, welche durch Wasserkraftanlagen in Kombination mit einem Umrichter zur Netzkupplung verfügbar ist, prinzipiell erörtert. Es wird das Systemkonzept dargestellt und auf die technischen Grenzen eingegangen. Abschließend wird noch ein mögliches Marktkonzept für die Systemdienstleistung "Momentanreserve" vorgestellt.
\end{abstract}

Schlüsselwörter: Momentanreserve; synthetische Schwungmasse; Wasserkraftanlagen

\section{Providing synthetic inertia with hydro power plants.}

The availability of a sufficient amount of inertia is important to guarantee stability of the electric power system. Nowadays, this inertia is provided by grid-synchronous rotating masses. In the context of this background the provision of synthetic inertia provided by hydro power units is investigated. Such units provide additional inertia by means of a converter coupling the units to the grid. The system concept of such units is introduced and the technical limitations are discussed. Finally, a possible market approach for the ancillary service "inertia" is presented.

Keywords: virtual inerta; hydro power; system stability

Eingegangen am 30. Juli 2016, angenommen am 29. September 2016, online publiziert am 5. Dezember 2016

\section{Einleitung}

\subsection{Bedeutung der Momentanreserve im elektrischen} Energiesystem

Mit dem Voranschreiten dezentraler und regenerativer Erzeugung in den europäischen Netzen sind auch die sogenannten Systemdienstleistungen immer mehr in Vordergrund gerückt. Darunter versteht man Maßnahmen, welche zusätzlich zum eigentlichen Energietransport ergriffen werden müssen, damit das Energienetz "funktioniert". Wichtige Beispiele dafür sind die Frequenzhaltung, die Spannungshaltung sowie die Sicherstellung des erlaubten Ausnutzungsgrads der Betriebsmittel. Die Verantwortung für den stabilen Netzbetrieb liegt beim Übertragungsnetzbetreiber (TSO, transmission system operator). Die physikalische Bereitstellung vieler Systemdienstleistungen geschieht jedoch durch Erzeugungsanlagen, auf welche der TSO seit der Strommarktliberalisierung keinen direkten Zugriff mehr hat. Die Erzeugungseinheiten stellen als wichtigste Systemdienstleistung Regelleistung zur Sicherstellung der Frequenzhaltung sowie Blindleistung für die Spannungshaltung zur Verfügung, wobei Regelleistung bzw. Regelenergie seit längerem auf einem entsprechendem Markt gehandelt wird, Blindleistung wie andere Systemdienstleistungen auch jedoch bisher in den meisten Ländern noch nicht abgegolten werden.

Wir beschäftigen uns im Folgenden mit einer weiteren wichtigen Systemdienstleistung, welche der Frequenzhaltung dient, die aber im Gegensatz zur Regelleistung (Primär-, Sekundär-, Tertiärreserve) noch nicht gehandelt oder abgegolten wird: der Schwungmasse als Lieferant von Momentanreserve. Im Falle eines plötzlichen Leistungsungleichgewichts ist es die Schwungmasse, $d$. h. die in den rotierenden Massen der direkt mit dem Netz verbundenen Maschinen ge- speicherte Rotationsenergie, welche einer unmittelbaren Frequenzänderung entgegenwirkt. Dies ist in Abb. 1 veranschaulicht: In den ersten Sekunden nach einem Leistungsdefizit, z. B. verursacht durch den Ausfall eines Kraftwerksblocks, sinkt die Netzfrequenz zunächst ab. Nach einigen Sekunden greift die Primärregelung, es wird zusätzliche Leistung ins Netz gespeist und ein weiteres Absinken der Frequenz verhindert. Die Sekundärregelung führt die Frequenz danach wieder auf ihren Sollwert von $50 \mathrm{~Hz}$ zurück und wird in weiterer Folge noch von der Tertiärregelung abgelöst, welche die Sekundärregelreserven wieder freigibt. Die prinzipielle Abhängigkeit des Verlaufs der Netzfrequenz von der Schwungmasse ist ebenfalls dargestellt: Umso größer die im Netz vorhandene Schwungmasse ist,

- desto geringer ist der Frequenzgradient $\mathrm{d} f / \mathrm{d} t$ und

- desto geringer ist die maximale Abweichung von der Nennfrequenz $\Delta f_{\max }$.

Als Maß für die Schwungmasse wird in Abb. 1 und im Folgenden die Trägheitskonstante $H$, gemessen in Sekunden s bzw. in MJ/MVA, verwendet. Diese ist definiert als

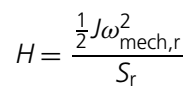

Schürhuber, Robert, ANDRITZ HYDRO GmbH, Eibesbrunnergasse 20, 1120 Wien Österreich (E-Mail: robert.schuerhuber@andritz.com); Lechner, Alois, ANDRITZ HYDRO GmbH, Eibesbrunnergasse 20, 1120 Wien, Österreich (E-Mail: alois.lechner@andritz.com); Gawlik, Wolfgang, Technische Universität Wien, Institut für Energiesysteme und Elektrische Antriebe, Gußhausstraße 25/ E370-1, 1040 Wien, Österreich (E-Mail: wolfgang.gawlik@tuwien.ac.at) 


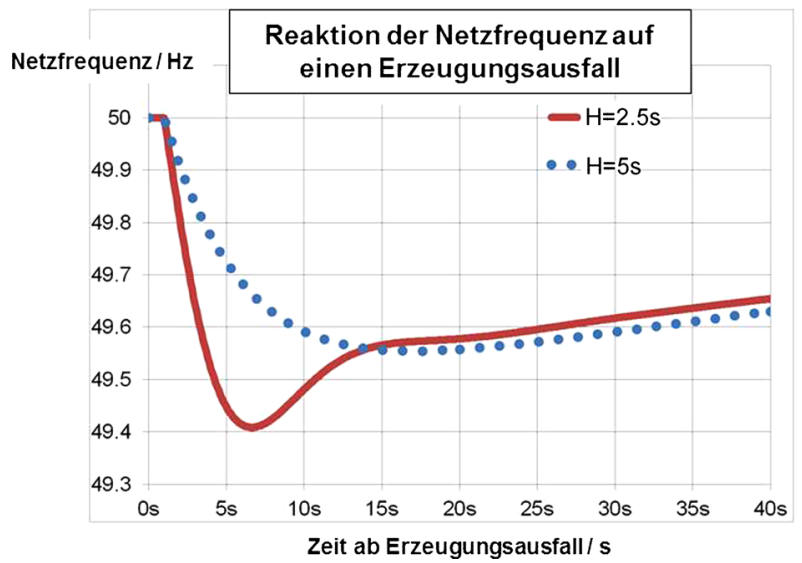

Abb. 1. Veranschaulichung des Zeitverlaufs der Netzfrequenz bei einem Erzeugungsausfall für unterschiedliche Werte der Momentanreserve, charakterisiert durch die Trägheitskonstante $\boldsymbol{H}$

mit dem Trägheitsmoment $J$ in $\mathrm{kg} \mathrm{m}^{2}$, der mechanischen Winkelgeschwindigkeit $\omega_{\text {mech, }}=2 \pi n / 60$ in s $^{-1}$ mit der Bemessungsdrehzahl $n$ in Upm und der Bemessungsscheinleistung $S_{r}$ der Maschine in VA. $H$ besitzt somit die Einheit Sekunden und entspricht der auf die Bemessungsscheinleistung bezogenen Rotationsenergie des Systems. Damit zeigt sich auch der Vorteil der Einheitenbezeichnung MJ/MVA, da hier klar ersichtlich ist, dass es sich um ein elektrisches $\mathrm{Maß}$ für die Schwungmasse handelt. Somit sind Verwechslungen mit der mechanischen Trägheitskonstante, welche auf die Wellenleistung bezogen ist, ausgeschlossen. Mit der in der Literatur ebenfalls vielfach verwendeten Anlaufzeitkonstante $T_{\mathrm{A}}$ besteht der $\mathrm{Zu}$ sammenhang $H=\frac{T_{A}}{2}$, wobei hier ebenfalls vorausgesetzt wird, dass die Rotationsenergie auf die Bemessungsscheinleistung bezogen ist.

Abbildung 1 zeigt den deutlichen Einfluss der Trägheitskonstante auf das Systemverhalten bei Störungen. Das Frequenzband für einen unbeeinflussten Netzbetrieb liegt zwischen $49 \mathrm{~Hz}$ und $51,5 \mathrm{~Hz}$, darunter kommt es zu Lastabwürfen bzw. darüber zur Netztrennung von Maschinensätzen [1, 2]. Würde nicht entsprechend reagiert, so besteht die Gefahr einer Instabilität, $d$. h. weicht die Netzfrequenz zu weit vom Nennwert ab, so existiert kein stabiler Betriebspunkt mehr und das System kollabiert. Für den Frequenzgradienten findet man in der Literatur Werte im Bereich von $0,5 \mathrm{~Hz} / \mathrm{s}$ bis $4 \mathrm{~Hz} / \mathrm{s}$, wobei der größere Wert für schwächere Netze gültig ist (z. B. Irland, Großbritannien, Türkei ohne Anbindung ans kontinentaleuropäische Verbundnetz). Die ausreichende Verfügbarkeit von Momentanreserve ist also essentiell für die Aufrechterhaltung der elektrischen Energieversorgung im Falle von Großstörungen.

\subsection{Aktuelle Entwicklung der Trägheitskonstante im europäischen Netz}

Die wirksame Trägheitskonstante des Netzes hängt von der Summe der am Netz angeschlossenen rotierenden Massen ab, $H=$ $\sum_{i=1}^{N} H_{i} \frac{S_{i}}{S_{\text {gesamt }}}$. Hierin ist $H$ die wirksame Gesamtträgheitskonstante, $N$ bezeichnet die Anzahl der angeschlossenen rotierenden Einheiten, $H_{i}$ und $S_{i}$ bezeichnen die Werte der Einheit $i$ und $S_{\text {gesamt }}$ ist die Summe der Scheinleistungen aller in die Summe eingehenden Einheiten [3]. Es handelt sich also um eine gewichtete Summe der Einzelträgheitskonstanten mit der auf die Gesamtscheinleistung bezogenen Scheinleistung als Gewichtungskoeffizient. Eine Voraussetzung für diese Näherung ist ein gut vermaschtes Netz, wie es im europäischen Verbundsystem vorhanden ist. Der Zusammenhang zwischen Leistungsbilanz und Netzfrequenz ist durch den Drallsatz

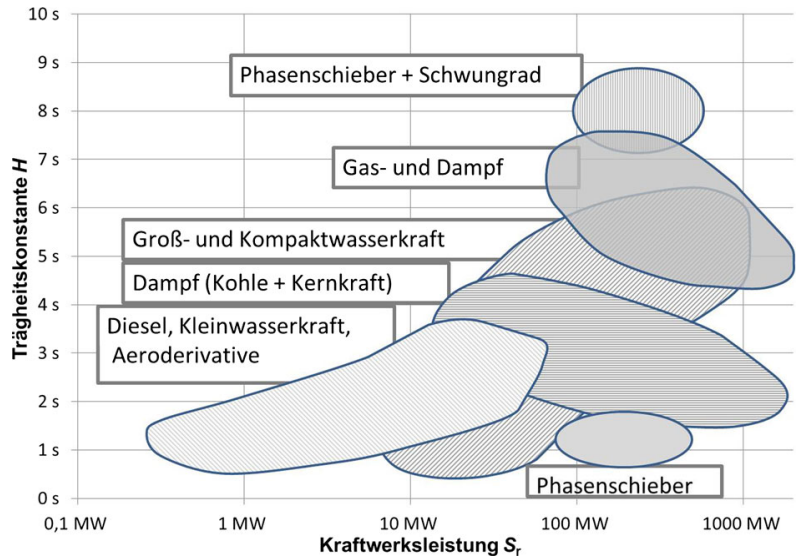

Abb. 2. Trägheitskonstante in Abhängigkeit der typischen Bemessungsscheinleistung $S_{r}$ einer Einheit

gegeben, der im Zusammenhang mit Stabilitätsuntersuchungen im Netz üblicherweise in der genäherten Form

$$
\mathrm{ROCOF}=\frac{\Delta P \cdot 50 \mathrm{~Hz}}{2 \cdot S_{\text {gesamt }} \cdot H}
$$

dargestellt wird. Darin bedeuten ROCOF $=\mathrm{d} f / \mathrm{d} t$ den Frequenzgradienten (rate of change of frequency) mit der Einheit $\mathrm{Hz} / \mathrm{s}, \Delta P=$ $P_{\text {mech }}-P_{\mathrm{el}}$ das Leistungsbilanzdefizit zwischen Erzeugung und Verbrauch, $S_{\text {gesamt }}$ die Summe aller angeschlossenen Scheinleistungen und $H$ die Gesamtträgheitskonstante des Systems. Hierbei wurde der Selbstregeleffekt der Last vernachlässigt sowie eine Linearisierung um den Punkt $f=50 \mathrm{~Hz}$ vorgenommen. Um einen Überblick über die Größenordnung der Trägheitskonstante verschiedener Erzeuger zu bekommen, betrachten wir zunächst die einzelnen Einheiten in Abhängigkeit vom Kraftwerkstypdargestellt in Abb. 2. Hier ist auf der Abszisse der typische Bereich der Bemessungsscheinleistung aufgetragen und auf der Ordinate ein üblicher Bereich der dazugehörigen Trägheitskonstanten. Es ist ersichtlich, dass größere, direkt über Synchronmaschinen ans Netz gekoppelte Einheiten eine Trägheitskonstante von etwa 3...6 s besitzen. Die relativ hohe Trägheitskonstante von Wasserkraftwerken rührt zum Großteil vom hohen Trägheitsmoment der als Schenkelpolmaschinen ausgeführten Generatoren her. Diese tragen etwa 90 \% zur Trägheitskonstante bei (der Rest verteilt sich auf Turbine, Welle und der im Laufrad mitrotierenden Wassermasse). Im Gegensatz dazu verhalten sich umrichtergekoppelte Erzeugungseinheiten, also hauptsächlich Windkraftanlagen und Photovoltaikanlagen, wie Anlagen mit Trägheitskonstante Null. Der Grund ist im Umrichter zu sehen, welcher die Erzeugungseinheit vom Netz entkoppelt und so die Änderungsrate der Drehzahl des Maschinensatzes von der Änderungsrate der Netzfrequenz unabhängig macht. PV-Anlagen verfügen inhärent über keine Schwungmasse. Für eine schwungmassenähnliche Reaktion müssen die Erzeugungseinheiten eine angepasste Regelung und einen ausreichend großen Energiespeicher besitzen, siehe Abb. 3 und Abschn. 2.1. Windkraftanlagen besitzen zwar Schwungmasse, diese reagiert üblicherweise wegen der Netzanbindung über Umrichter nicht auf Netzfrequenzänderungen. Damit kann folgender Schluss für die Systemträgheitskonstante gezogen werden: Je mehr konventionelle, direkt über Synchronmaschinen ans Netz gekoppelte Erzeugungseinheiten durch umrichtergekoppelte Einheiten ersetzt werden, desto geringer wird die Gesamtträgheitskonstante des Systems. Durch die Volatilität dieser Einheiten wird diese auch stark zeitabhängig und schwankt in einem relativ großen Bereich, 


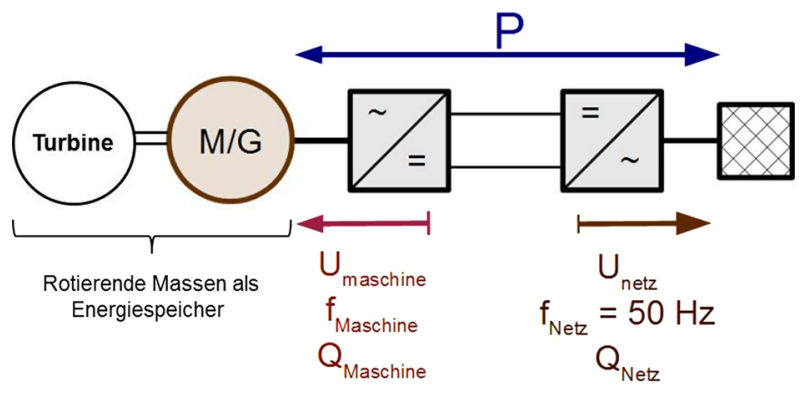

Abb. 3. Konzeptionelle Darstellung umrichtergekoppelter Anlagen mit rotierenden Massen als Speicher von Rotationsenergie. Der Umrichter entkoppelt das Netz von der rotierenden Maschine und macht so die ins Netz abgegebene Leistung innerhalb anlagenabhängiger Grenzen unabhängig von der Maschinendrehzahl

für Deutschland beispielsweise etwa zwischen 3 s und 6 s [4]. Nun ist im europäischen Verbundnetz aller Voraussicht nach auch mittelfristig in Summe genügend Momentanreserve vorhanden, um einen zu großen Frequenzgradienten zu verhindern [5]. Dabei muss allerdings festgestellt werden, dass dies auf Eigenschaften der speisenden konventionellen Erzeugungsanlagen basiert, die derzeit nicht regulatorisch eingefordert werden und auch nicht vergütet werden. Die Stabilität des Systems wäre nicht in allen Betriebszuständen gewährleistet, wenn ausschließlich die derzeitigen regulatorischen Mindestanforderungen eingehalten würden. Besonders kritisch ist die Situation im Fall von Großstörungen wie im Jahr 2006, bei der das ENTSO-E Continental Europe-Netz in 3 Teilnetze zerfallen ist [6]. Wenn in einem dieser Teilnetze zu wenig Momentanreserve vorhanden ist (z. B. bei hohem Anteil an Erzeugung durch Windkraft oder Photovoltaik), so läuft dieses in Gefahr, seine Stabilität zu verlieren.

\section{Bereitstellung synthetischer Schwungmasse durch Wasserkraftwerke}

\subsection{Bereitstellung synthetischer Schwungmasse durch umrichtergekoppelte Anlagen}

Wie in Abschn. 1.2 dargelegt ist bei umrichtergekoppelten Anlagen die Kopplung von Frequenzgradient und eingespeister Leistung aufgehoben. Die Grundidee bei der Bereitstellung synthetischer Schwungmasse ist es nun, diese Kopplung mit Hilfe einer entsprechend entworfenen Regelung wiederherzustellen. Die Regelung reagiert also auf den Verlauf der Netzfrequenz und fordert davon abgeleitet elektrische Leistung an. Diese kann vom Umrichter sehr rasch bereitgestellt werden. Die Leistungsänderung auf der Netzseite muss energetisch durch eine entsprechende Energieänderung auf der Erzeugerseite gedeckt sein. Dies bedeutet, dass Systeme, welche synthetische Schwungmasse bereitstellen sollen, zusätzlich zur pas- senden Regelung zwingend einen Energiespeicher enthalten müssen. Im Falle von Wasserkraftanlagen und Windenergieanlagen ist der Energiespeicher die rotierende Masse, es sind aber auch andere Formen der Speicherung denkbar, etwa Batterien oder Kondensatoren. Für eine Abschätzung der Energiemenge, welche in einer Wasserkraftanlage in Form von Rotationsenergie ein- bzw. ausgelagert werden kann, nehmen wir eine zulässige Drehzahlabweichung um $15 \%$ von der Nenndrehzahl an. Damit stehen bei einer typischen 25 MVA Maschine eines Laufkraftwerks etwa 10..30 MJ und bei einem 250 MVA Speicherkraftwerk etwa 300 MJ an Rotationsenergie zur Verfügung, welche zur Bereitstellung synthetischer Schwungmasse genutzt werden kann.

Ein Vorteil von umrichtergekoppelten Anlagen, welche synthetische Schwungmasse bereitstellen, ist, dass man hier nicht an die durch die rotierenden Massen definierte Trägheit gebunden ist. Vielmehr kann man im Rahmen der anlagenbedingten Grenzen einen beliebigen, passenden, zeitlichen Verlauf der Trägheitskonstante vorgeben. So ist es beispielsweise möglich, die Schwungmasse größer als die reale Schwungmasse erscheinen zu lassen oder die Trägheitskonstante zeitlich in Abhängigkeit von Frequenzabweichung, Frequenzgradient oder deren Vorzeichen einzustellen. Ein Schema des beschriebenen Prinzips ist in Abb. 4 dargestellt.

In welchem Maß die Erzeugungseinheiten durch synthetische Schwungmasse zur Bereitstellung von Momentanreserve beitragen können, hängt von den systemtechnischen Rahmenbedingungen ab. Es gilt verschiedene technische Grenzen der Anlage nicht zu verletzen. Von elektrischer Seite betrifft dies vor allem den Umrichter, welcher im Gegensatz zu elektrischen Maschinen kaum überlastfähig ist. Als Richtwert für die maximale Leistung kann die Bemessungsleistung des Umrichters angenommen werden, je nach Auslegung ist eine Überlast um etwa den Faktor 1,5 für ein bis zwei Sekunden denkbar. Die elektrische Leistungsreserve des aktuellen Arbeitspunkts gegenüber dem Bemessungspunkt sowie die kurzfristige Überlastfähigkeit des Umrichters bestimmen also das zulässige $\mathrm{Maß}$ an synthetischer Schwungmasse. Nehmen wir beispielsweise einen Faktor 3 für die synthetische Schwungmasse gegenüber der realen Schwungmasse einer synchronen Einheit mit einer Trägheitskonstanten $H$ von $5 \mathrm{MJ} / \mathrm{MVA}$ sowie einen Frequenzgradienten von $0,5 \mathrm{~Hz} / \mathrm{s}$ an, so ergibt sich gemäß Gl. (1) eine zusätzliche Leistungseinspeisung von $\Delta p=\frac{\Delta P}{S_{r}}=\frac{2 \cdot 3 \cdot H \dot{f}}{f}=0,3$ pu. Weitere Einschränkungen existieren auf der mechanischen bzw. der hydraulischen/aerodynamischen Seite. Eine plötzliche Änderung der elektrischen Leistung bewirkt einen Momentenstoß, welcher vom mechanischen System beherrscht werden muss. Bei Wasserkraftanlagen hat dies auch einen Druckstoß im hydraulischen System zur Folge, auch dieser muss innerhalb der Auslegungswerte der Anlage bleiben. Bei Windkraftanlagen ist vor allem das Schwingungsverhalten der Anlage bei solch einer Anregung zu untersuchen. Diese grundsätzlichen Überlegungen zeigen, dass die Bereitstellung syn-

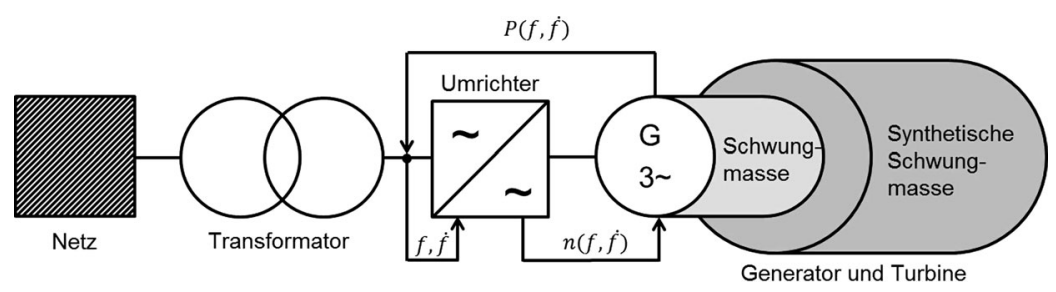

Abb. 4. Schematische Darstellung der Bereitstellung synthetischer Schwungmasse durch Erzeugungseinheiten mit rotierenden Massen. Die durch den Umrichter aufgehobene Kopplung zwischen Netzfrequenz und Maschinendrehzahl wird durch eine intelligente Regelung wiederhergestellt. Dadurch kann flexibel Zusatzleistung in Abhängigkeit vom Verlauf der Netzfrequenz bereitgestellt werden 
Pumpspeicherkraftwerk mit doppeltspeisendem Motor-Generator

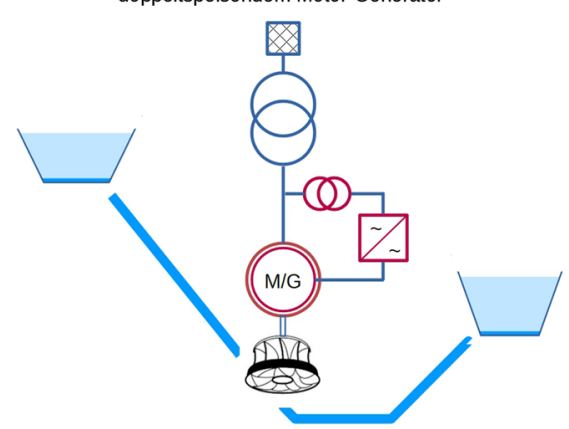

Pumpspeicherkraftwerk mit Vollumrichter

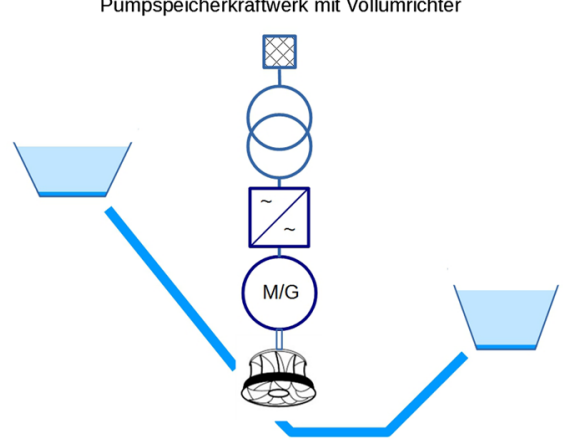

Laufwasserkraftwerk mit Vollumrichter

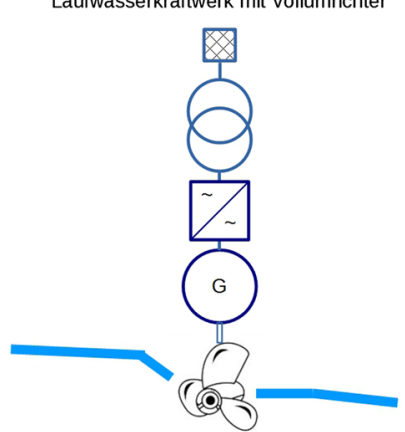

Abb. 5. Schematische Darstellung verschiedener Konfigurationen von Wasserkraftanlagen, welche synthetische Schwungmasse bereitstellen können

thetischer Schwungmasse je nach Ausführung und Arbeitspunkt unterschiedliche Auswirkungen auf den Antriebsstrang sowie die Mechanik der Erzeugungsanlage hat und jedenfalls zu untersuchen ist.

\subsection{Besonderheiten von Wasserkraftanlagen}

Wasserkraftanlagen können in verschiedener Weise als Quellen synthetischer Schwungmasse dienen. Zurzeit existieren nur wenige Anlagen, welche über einen Umrichter ans Netz gekoppelt sind. Es handelt sich hierbei vor allem um große Pumpspeicherkraftwerke, der Umrichter dient zur Drehzahleinstellung, was Vorteile beim Anlagenbetrieb bringt. Hauptsächlich wird durch eine variable Drehzahl die Flexibilität der Anlage bezüglich ihres Arbeitsbereiches stark erhöht sowie ihre dynamische Reaktion verbessert. Prinzipiell existieren, ähnlich wie bei Windkraftanlagen, zwei verschiedene Konzepte drehzahlvariabler Pumpspeicher: Anlagen mit Vollumrichter sowie solche mit doppelspeisendem Motor-Generator. Für die Bereitstellung synthetischer Schwungmasse sind Vollumrichteranlagen wesentlich besser geeignet, da Anlagen mit doppelspeisendem MotorGenerator bedingt durch den Umrichter im Rotorkreis harte Drehzahlgrenzen besitzen, welche zwingend eingehalten werden müssen. Dadurch ist arbeitspunktanhängig eine Beschränkung der zusätzlich zur Verfügung stehenden Rotationsenergie gegeben. Anlagen mit Vollumrichter erlauben den Betrieb in einem weiten Drehzahlbereich, von der elektrischen Seite her sind dabei praktisch keine Einschränkungen vorhanden. Auch auf der hydraulischen Seite kann eine große Drehzahlvarianz ausgenutzt werden. Es existieren zwar Einschränkungen auf der hydraulischen Seite, aber ein kurzfristiger Betrieb im Sekundenbereich außerhalb des stationären Arbeitsbereichs bedeutet keine wesentliche Belastung für die Maschine und bewirkt im Wesentlichen einen etwas erhöhten Verschleiß. Da es sich bei der Bereitstellung von synthetischer Schwungmasse in hohem Ausmaß jedoch um einen seltenen Betriebszustand handelt, ist hierbei keine relevante Verringerung der Maschinenlebensdauer zu erwarten.

Die Vollumrichterlösung wäre auch die geeignete Variante, um bestehende Laufkraftwerke umzurüsten, damit diese synthetische Schwungmasse als Systemdienstleistung anzubieten können. Laufkraftwerke werden meistens sehr nahe am Dauerbetrieb gefahren, die zusätzlich verfügbare Momentanreserve steht daher verlässlich zur Verfügung. Wird der Umrichter nicht benötigt, so kann ein Bypassschalter vorgesehen werden, um in diesem Fall keinen Wirkungsgradverlust durch den Umrichter zu erleiden. In Abb. 5 sind die verschiedenen erwähnten Varianten als Übersicht dargestellt.

Durch die großen vorhandenen Drehzahlreserven von Wasserkraftanlagen ist also bei geeigneter Auslegung des Umrichters eine

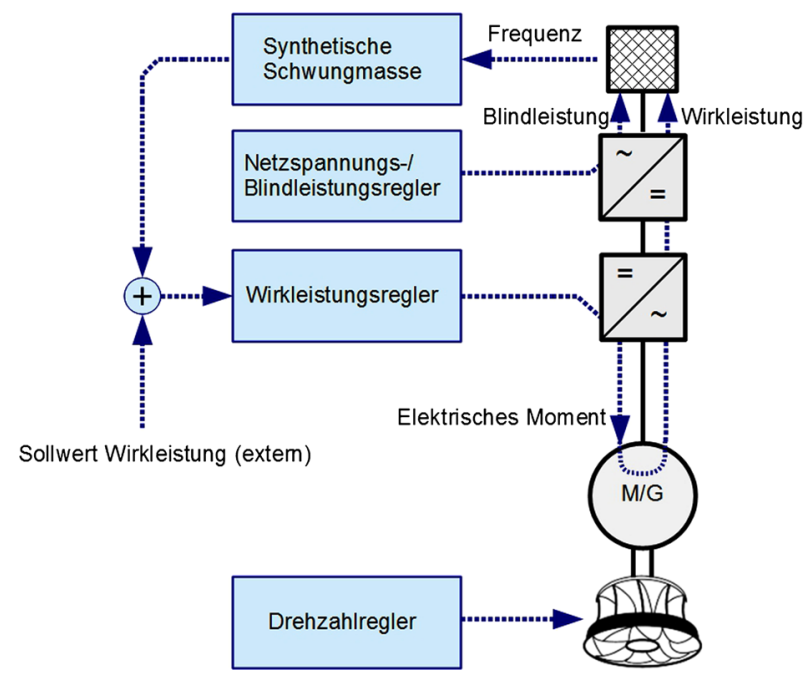

Abb. 6. Überblick der Regelungsstruktur für synthetische Schwungmasse von Wasserkraftanlagen

große synthetische Schwungmasse realisierbar, sie sind somit vorteilhaft für die Bereitstellung dieser Systemdienstleistung geeignet.

\section{Simulation des Verhaltens von Wasserkraftanlagen}

Der Einsatz von Umrichtern in Wasserkraftanlagen führt einen zusätzlichen Freiheitsgrad ein, nämlich die Drehzahl des Maschinensatzes. Auf die Drehzahl wirken sowohl das elektrische Moment, das der Generator aufgrund der vom Umrichter eingestellten Stromund Spannungswerte entwickelt, als auch das Antriebsmoment der Turbine aufgrund der Stellung des Turbinen-Stellapparats und der aktuell herrschenden hydraulischen Verhältnisse.

\section{1 Übersicht über die verwendete Regelungsstruktur}

Als Stellgrößen für eine drehzahlvariable Wasserkraftanlage sind das elektrische Moment des Generators und die Stellung(en) des Turbinen-Leitapparats günstig, um die beiden Systemgrößen Drehzahl und elektrische Leistung am Netzübergabepunkt zu regeln. Im Prinzip können beide Stellgrößen beide Systemgrößen beeinflussen, daher gibt es keine vorgegebene Zuordnung der Stellgrößen in der Regelungsstruktur. Um einen möglichst dynamischen Verlauf der elektrischen Leistung zu gewährleisten, ist die Zuordnung des Generatordrehmoments als Ausgang des Leistungsreglers erforderlich. Die Drehzahl wird somit über den Turbinen-Stellapparat eingeregelt. Abbildung 6 zeigt die Struktur eines solchen Konzepts. 


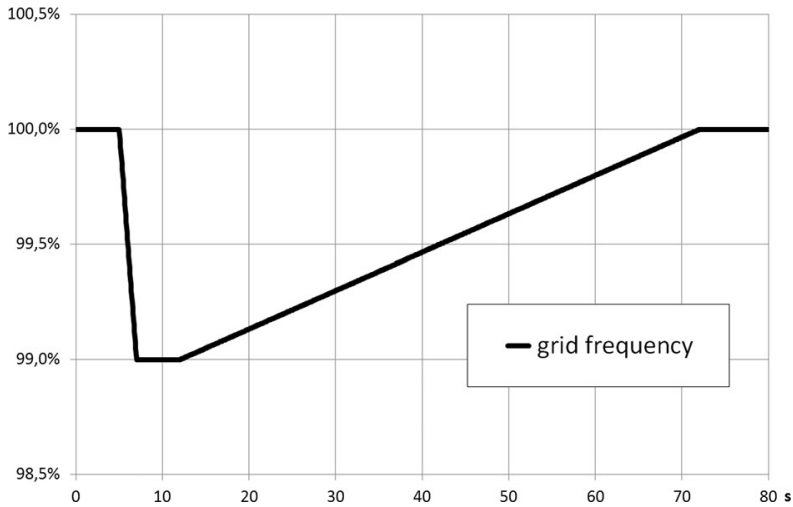

Abb. 7. Netzfrequenzverlauf, aus Geradenstücken zusammengesetzt

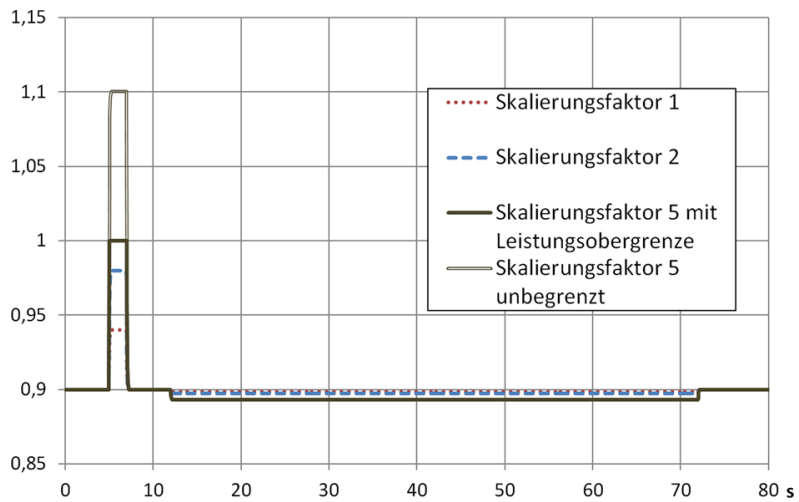

Abb. 8. Simulierter Leistungsverlauf am Netzanschlusspunkt mit Skalierungsfaktor 1, 2 und 5 bei konstruiertem Netzfrequenzverlauf

Die Bereitstellung von synthetischer Schwungmasse erfolgt in dieser Regelungsstruktur, indem zu dem betriebsmäßig vorgegebenen Leistungssollwert zusätzlich der Leistungsanteil der synthetischen Schwungmasse, berechnet anhand der Netzfrequenz, der Netzfrequenzänderung und der Schwungmassenskalierung, über die Drehmomentregelung des Generators eingeregelt wird.

3.2 Einfluss verschiedener Parameter auf das Systemverhalten Die wesentlichen Parameter, die auf das Systemverhalten Einfluss nehmen, sind der Skalierungsfaktor der Schwungmasse und die stationäre Drehzahl. Zusätzlich ist der Abstand des stationären Arbeitspunktes von den Systemgrenzen von Belang, daraus leitet sich die Obergrenze der verfügbaren Stützleistung bzw. Stützenergie ab. In Abb. 8 wird exemplarisch der Leistungsverlauf für die Schwungmassenskalierungen 1, 2 und 5 bei einem aus Geradenstücken zusammengesetzten Netzfrequenzverlauf (Abb. 7) gezeigt. Eine Schwungmassenskalierung bedeutet in diesem Zusammenhang eine gegenüber der inhärent vorhandenen Schwungmasse (Abb. 2) eine um den Skalierungsfaktor erhöhte synthetische Schwungmasse. Der stationäre Arbeitspunkt ist bei $90 \%$ der Nennleistung angenommen, die Grenze der Netzleistung mit $100 \%$. Es zeigt sich, dass bei der Schwungmassenskalierung 5 die Netzleistungsgrenze überschritten würde und daher der gewünschte Wert der Netzleistung nicht eingeregelt werden kann.

Der Kurzzeitspeicher zur Implementierung der synthetischen Schwungmasse ist die physikalische Schwungmasse des Wasserkraftwerks. Dementsprechend bildet sich eine Netzfrequenzänderung mit dem jeweiligen Skalierungsfaktor umgerechnet als Drehzahländerung $a b$, also wird bei einer Frequenzabsenkung um $1 \%$

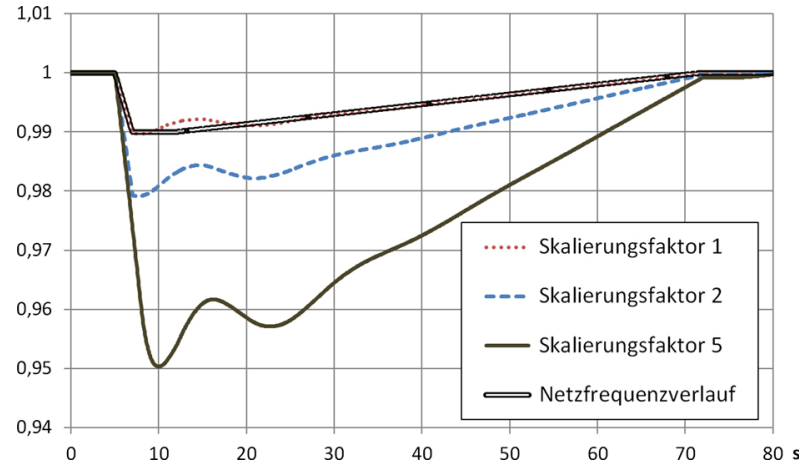

Abb. 9. Simulierter Drehzahlverlauf mit Skalierungsfaktor 1, 2 und 5 bei konstruiertem Netzfrequenzverlauf

bei einem Skalierungsfaktor 1 auch die Drehzahl um $1 \%$ absinken, analog zu einer Anlage ohne Drehzahlregelmöglichkeit. Ein höherer Skalierungsfaktor bewirkt natürlich eine entsprechenden höhere Folgereaktion. Diese Überlegung ist in Abb. 9 quantitativ dargestellt, sie zeigt den zum Leistungsverlauf in Abb. 8 gehörenden Verlauf der Drehzahl. Die Auswirkungen dieser Drehzahlvariation auf das mechanische und hydraulische System sind gesondert zu untersuchen und zu bewerten [7]

\section{Synthetische Schwungmasse als vergütete Systemdienstleistung}

Im Abschn. 1.1 wurde bereits ausgeführt, dass Schwungmasse und Momentanreserve von essentieller Bedeutung für die Stabilität des elektrischen Verbundsystems sind. Insofern haben sie auch einen eigenen Wert als Systemdienstleistung, aber derzeit in der Regel noch keinen Preis.

Eine Möglichkeit, ein Vergütungsschema für die Systemdienstleistung „Momentanreserve” zu etablieren, besteht darin, ein Regelleistungsprodukt zu definieren, dessen Bereitstellung zeitlich noch vor der Kette "Primärregelleistung" (PRL, oder "Frequency Containment Reserve" FCR) - "Sekundärregelleistung" (SRL, oder "Frequency Restoration Reserve" FRR) - Tertiärregelleistung (TRL, oder Replacement Reserve RR) angesiedelt ist, d. h. das unmittelbar nach einer auftretenden Frequenzabweichung bzw. durch Frequenzgradienten aktiviert wird und durch die Primärregelung abgelöst wird. Solche Ansätze werden z. B. als "Enhanced Frequency Response" durch National Grid in Großbritannien verfolgt.

Als weitere Möglichkeit soll in diesem Artikel das Konzept der „Inertia Certificates" präsentiert werden. Es basiert auf der folgenden grundsätzlichen Überlegung: Angenommen, für ein Synchrongebiet kann eine minimal notwendige Trägheitskonstante definiert werden, die für den stabilen Netzbetrieb vorausgesetzt werden muss. Hierbei ist festzuhalten, dass das Synchrongebiet nicht mit einer Regelzone ident sein muss, aber eine möglichst gleichmäßige Verteilung der Schwungmasse im System anzustreben ist. Dann können Anlagen, die diese Anforderung bezüglich der Trägheitskonstante anteilig übererfüllen, die zusätzlich bereitgestellte Schwungmasse vermarkten und solchen Anlagen, die selber keine oder zu wenig Momentanreserve liefern, in Form von Inertia Certificates (IC) gegen eine Vergütung zur Verfügung stellen.

Zur Veranschaulichung des Konzeptes sei dabei von einem einfachen Beispielsystem ausgegangen, in dem eine schwungmassenbehaftete Anlage mit einer Bemessungsleistung von $S_{\text {inertiaprovider }}=$ 100 MVA und einer Trägheitskonstante von $H_{\text {inertiaprovider }}=4 \mathrm{~s}$ zur Lastdeckung beiträgt. Für das Beispielsystem soll eine minimale 
Netzanlaufzeitkonstante von $5 \mathrm{~s}$, d. h. eine minimale Trägheitskonstante von $H_{\min }=2,5 \mathrm{~s}$ wirksam sein. Die schwungmassenbehaftete Anlage übererfüllt diese Anforderung um

$$
\begin{aligned}
\mathrm{IC}_{\text {inertiaprovider }} & =S_{\text {inertiaprovider }} \cdot\left(H_{\text {inertiaprovider }}-H_{\text {min }}\right) \\
& =100 \mathrm{MVA} \cdot 1,5 \mathrm{~s}=150 \mathrm{MVAs} .
\end{aligned}
$$

Die überschüssige Schwungmasse kann also in Form von Inertia Certificates in Höhe von insgesamt 150 MVAs vermarktet und an Anlagen verkauft werden, die zu wenig oder gar keine Schwungmasse bereitstellen. Im Beispielsystem könnten das nach

$$
\mathrm{IC}_{\text {inertiaconsumer }}=S_{\text {inertiaconsumer }} \cdot\left(H_{\min }-H_{\text {inertiaconsumer }}\right)
$$

z. B. trägheitslose Einspeiser $\left(H_{\text {inertiaconsumer }}=0 \mathrm{~s}\right)$ mit einer Gesamtleistung von $S_{\text {inertiaconsumer }}=60$ MVA sein.

Darüber hinaus können aber auch Anlagen, die die Mindestanforderungen gerade erfüllen, oder solche, die ihre überschüssige Schwungmasse bereits vermarktet haben, in bestimmten Zeiträumen weitere Inertia Certificates herausgeben, sofern sie sich in diesen Zeiträumen im leistungsreduzierten Betrieb befinden, z. B. weil sie nur einen Teil ihrer Auslegungsleistung $S_{\text {inertiaprovider_partialload }}$ am Energiemarkt platzieren konnten. Diese zusätzlichen ICs ergeben sich aus

$I C_{\text {inertiaprovider_partialload }}=\left(S_{\text {inertiaprovider }}-S_{\text {inertiaprovider_partialload }}\right) \cdot H_{\min }$.

So können auch Anlagen, die wegen höherer Grenzkosten für die gelieferte Energie aus dem Markt gedrängt werden, einen Teil ihrer Mindereinnahmen kompensieren, während sich umgekehrt trägheitslose Anlagen über den Erwerb von ICs an den Kosten für die Systemdienstleistung Schwungmasse beteiligen.

Das Konzept setzt voraus, dass alle Erzeugungsanlagen regulatorisch zum Nachweis der Deckung der minimalen anteiligen Schwungmasse bezogen auf ihre Einspeiseleistung verpflichtet werden. Es setzt weiters voraus, dass die Anlagen, die ICs zur Verfügung stellen, in den vereinbarten Zeiträumen auch am Netz sind. Deswegen ist eine Koordination des Handels mit ICs mit den entsprechenden Fahrplänen der Anlagen notwendig.

Als Alternative zum Erwerb von ICs durch trägheitslose bzw. trägheitsarme Erzeugungsanlagen besteht die Möglichkeit, diese Anlagen mittels einer Ergänzung durch Energiespeicher, z. B. Supercaps im DC-Kreis von Photovoltaikanlagen oder Batterien und einer entsprechende Regelung der Wechselrichter, die synthetische Schwungmasse emuliert, zu erweitern. Es sei an dieser Stelle darauf hingewiesen, dass die Kapazität dieser Energiespeicher nicht identisch mit der Höhe der zu erwerbenden ICs ist. Das liegt daran, dass die ICs sich im vorgestellten Konzept aus der gesamten in der zu vermarkteten Schwungmasse gespeicherten Energie bestimmen, während nachzurüstende Energiespeicher nur die Kapazität zur Verfügung stellen müssen, um die Energie aufzunehmen bzw. abzugeben, die zwischen den Frequenzen mit dem Netz ausgetauscht wird, bei denen sich die Erzeugungsanlagen nicht vom Netz trennen sollen, d. h. von $47,5 \mathrm{~Hz}$ bis $51,5 \mathrm{~Hz}$.

Es ist dennoch davon auszugehen, dass der Erwerb von ICS zunächst, insbesondere solange noch ein ausreichendes Angebot an ICS durch die im Netz befindlichen "konventionellen" Anlagen besteht, die kostengünstigere Variante darstellt. Erst wenn diese Quellen von ICs erschöpft sind, wird das Anbieten von zusätzlicher Schwungmasse durch eigens dafür vorgesehene Anlagen wie Schwungradspeicher oder Phasenschieber mit zusätzlicher Schwungmasse ein valides Geschäftsmodell. Ein Konzept dieser Art ist auch kompatibel mit den Eigenschaften von Wasserkraftanlagen: Laufwasserkraftwerke sind meist am Netz und können ihre Schwungmasse daher dauernd zur Verfügung stellen. Pumpspeicherkraftwerke erlauben einen Phasenschieberbetrieb, in dem Schwungmasse verlustarm verfügbar ist. Durch zusätzliche synthetische Schwungmasse können diese Anlagen einen zusätzlichen Ertrag erwirtschaften, speziell wenn in ein zukünftiges Handelsmodell ein Faktor für instantan eingespeiste Leistung eingebracht wird.

\section{Zusammenfassung}

Die Momentanreserve gewinnt in einem zunehmend von der Einspeisung durch erneuerbare Energien über Umrichter geprägten Netz immer mehr an Bedeutung. Ein gewisses Maß an Momentanreserve ist für die Systemstabilität nötig, praktisch verringert sich der Abstand zu diesem Grenzwert durch eine Erzeugungsstruktur, welche standardmäßig oftmals keine Schwungmasse besitzt und daher nicht zur Momentanreserve beiträgt (die meisten Windkraftanlagen, Photovoltaikanlagen). Wasserkraftanlagen, welche auch zukünftig als fixer Bestandteil des Erzeugungsmix am Netz sind, können vorteilhaft zur Bereitstellung von Momentanreserve eingesetzt werden. Besonders flexibel werden sie durch Kombination mit einem Umrichter. Damit kann je nach Bedarf synthetische Schwungmasse zur Verfügung gestellt werden, welche die inhärent verfügbare Schwungmasse quantitativ übertreffen kann und auch in kürzerer Zeit verfügbar ist. Zukünftig ist eine Abgeltung der Systemdienstleistung Momentanreserve vorstellbar, um auch weiterhin die Systemstabilität in allen Betriebszuständen zu gewährleisten. Eine mögliche Grundidee wäre ein Handelsschema, in dem Anlagen ohne Schwungmasse durch Zukauf von Schwungmassezertifikaten den von innen geforderten Beitrag leisten.

Open Access This article is distributed under the terms of the Creative Commons Attribution 4.0 International License (http://creativecommons.org/ licenses/by/4.0/), which permits unrestricted use, distribution, and reproduction in any medium, provided you give appropriate credit to the original author(s) and the source, provide a link to the Creative Commons license, and indicate if changes were made.

Literatur

1. Technische und organisatorische Regeln für Betreiber und Benutzer von Netzen Teil E: Technische Maßnahmen zur Vermeidung von Großstörungen und Begrenzung ihrer Auswirkungen Version 2.12011

2. Verband der Netzbetreiber VDN e.V.: Netz- und Systemregeln der deutschen Übertragungsnetzbetreiber, Transmission Code 2007, Berlin, August 2007

3. Kundur, P. (1994): Power System Stability and Control. New York: McGraw-Hill.

4. Ulbig, A., Borsche, T. S., Andersson, G.: Impact of Low Rotational Inertia on Power System Stability and Operation. arXiv:1312.6435v4 [math.OC].

5. Deutsche Energie - Agentur GmbH (dena): Momentanreserve 2030. Bedarf und Erbringung von Momentanreserve 2030. Endbericht, Deutsche Energie-Agentur GmbH (dena), Berlin, 2016.

6. UCTE (2007): Final Report - System Disturbance on 4 November 2006.

7. Lechner, A., Hell, J., Schürhuber, R. (2014): Pumped Storage Contribution to Grid Inertia. In 18th International Seminar on Hydropower Plants, Laxenburg. 


\section{Autoren}

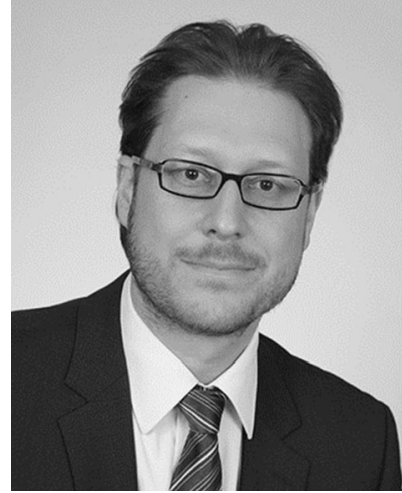

Robert Schürhuber

studierte Elektrotechnik und Technische Mathematik an der Technischen Universität Wien, Österreich, und promovierte dort 2003 im Gebiet der theoretischen Elektrotechnik. Seitdem war er in der Industrie in verschiedenen Gebieten der Energietechnik tätig und ist seit 2010 Fachexperte für elektrische Energietechnik bei der ANDRITZ HYDRO GmbH. Weiters ist er Vorsitzender des österreichischen Fachunterausschusses TSK H7 für Kurzschluss in elektrischen Anlagen, Mitglied der DKE/UK121.1 für Kurzschlussberechnung sowie Lektor an der Technischen Universität Wien.

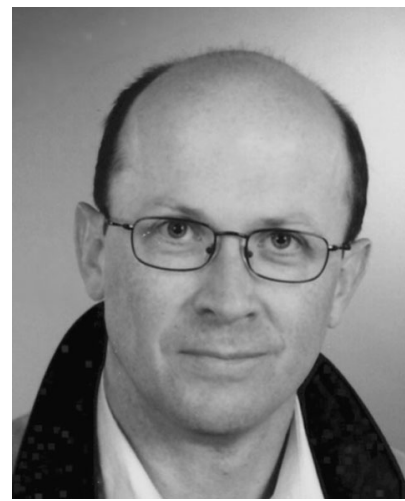

\section{Alois Lechner}

ist Systemtechniker für das elektrische Equipment von großen Wasserkraftanlagen bei ANDRITZ HYDRO GmbH in Wien, Österreich. Nach der Dissertation an der Technischen Universität Wien, Österreich, im Jahr 1991 war er bis 2011 im Bereich der hochdynamischen und hochgenauen Prüfstandsantriebe für VA TECH und für Schneider Electric beschäftigt. Seit März 2011 ist er bei ANDRITZ HYDRO mit dem Schwerpunktthema drehzahlvariable Energieerzeugung tätig.

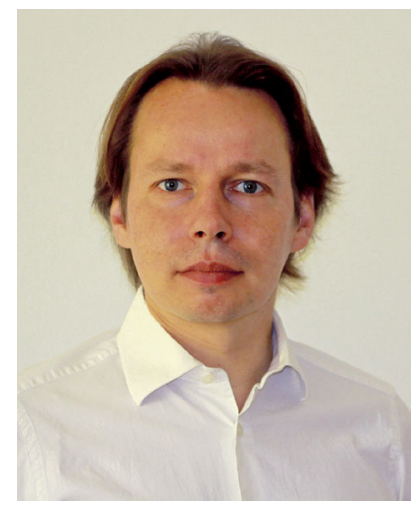

Wolfgang Gawlik

hat an der Friedrich-AlexanderUniversität (FAU) ErlangenNürnberg, Deutschland, Elektrotechnik mit Schwerpunkt Energietechnik studiert. Bis zu seiner Berufung als Universitätsprofessor für Energiesystemtechnik an der Technischen Universität Wien, Österreich, im Jahr 2011 war er als Senior Key Expert System Dynamics und Projektmanager bei Siemens Power Technologies International, Erlangen, tätig. 